\title{
LA SOMBRA DEL OTRO: UN ESTUDIO SOBRE EL DOBLE*
}

Flora Botton-Burlá

Una observación superficial parece sugerir que los dobles surgen con más frecuencia en la literatura que en la vida real, y que su aparición, las más de las veces, provoca miedo, horror, angustia o, por lo menos, un grave desasosiego. Pero no voy a hablar aquí de los dobles reales, de esos gemelos físicos o espirituales que todos, o casi todos, hemos conocido alguna vez, sino de aquellos que son producto de la imaginación del escritor.

La presencia de los dobles literarios plantea una serie de problemas de índole diversa: uno se pregunta de dónde vienen, cómo han surgido fuáles son los efectos que tiene su existencia en la vida de los demás y, sobre todo, en la vida de aquellos a quienes duplican o con quienes tienen esa especialísima relación.

Mi propósito aquí es tratar de desentrañar, por medio del estudio de algunos personajes dobles, cuáles son las características comunes que podrían servir para definirlos. He elegido para ello un cuento de Borges, "El otro"; dos cuentos de Cortázar, "Lejana" y "La noche boca arriba"; "William Wilson", de Edgar Allan Poe; "Monsieur du Miroir", de Hawthorne; y "The Dummy", de Susan Sontag. Cada uno de ellos presenta un tipo de doble diferente, pero todos comparten ciertas características fundamentales.

Para facilitar el estudio he llamado a los personajes, en todos los casos, 1 y 2: 1 es el primero, aquel que se enfrenta al hecho de que tiene un doble, el que Otto Rank, en su estudio clásico sobre el tema, llama "el individuo"; 2 es la copia, el segundo que aparece en escena, al que Rank llama "el doble". 1

Empezaré por decir unas cuantas palabras sobre los diferentes tipos de dobles que aparecen en los textos de los que me ocupo. Los dobles pueden ser:

- Este artículo es (con algunas ligeras modificaciones) el texto de una ponencia presentada en el décimo congreso de la Asociación Internacional de Literatura Comparada (Nueva York, agosto de 1982).

1 O. Rank, The Double, p. 18. 
a) Un solo individuo con dos personalidades o manifestaciones. El ejemplo clásico de este tipo es Dr. Jekyll and Mr. Hyde. Pero, por razones de espacio, no voy a hablar aquí de Stevenson, sino que me limitaré a los textos antes mencionados. Es el caso de "La noche boca arriba", de Julio Cortázar. En este cuento, el personaje al que llamo 1 es un motociclista que sufre un accidente, a consecuencia del cual es llevado al hospital y operado. Después de la operación tiene una serie de sueños extraños, en los que se ve transformado en un guerrero moteca perseguido por los aztecas en la guerra florida. Esta segunda personalidad, la del sueño, va adquiriendo fuerza progresivamente, hasta que termina por eliminar a la primera. Aquí el personaje tiene un solo cuerpo, aparentemente, y, también aparentemente, una sola conciencia, que se sabe doble. Sin embargo, para 1, sólo su conciencia es la real: sabe que es un motociclista que sueña que es un guerrero. Por su parte el guerrero, 2, no parece conocer la existencia de 1 y nunca se refiere a él. Hasta los últimos instantes del cuento, el lector sabe que ve las cosas a través de la conciencia de 1 , y que 2 sólo es un producto del delirio de éste.

b) Otra forma que puede asumir el doble es la de dos seres independientes, pero con una relación especial entre sí. Esto ocurre en "Lejana" (también de Cortázar), donde 1 (una chica de sociedad, que vive en Buenos Aires) tiene la conciencia de que existe otra mujer, a la que nunca ha visto y de la cual ignora todo, hasta su nombre, pero con la que tiene una extraña afinidad de sensaciones. Sólo sabe que la otra vive en una ciudad que está muy lejos de donde ella se encuentra (más tarde sabremos que esta ciudad es Budapest). La relación entre 1 y 2 se establece, pues, sin que haya contacto físico ni tonocimiento directo, a través de una gran distancia, y es necesario que ésta desaparezca para que la situación pueda concretarse y llegar a su clímax. En "William Wilson" también tenemos dos personas independientes, pero fisicamente idénticas (aunque esta identidad sólo parece ser perceptible para 1); ellos sí se conocen, e incluso son compañeros de clase. Son personajes independientes, asimismo, los dobles que aparecen en "The Dummy", pero de ellos hablaremos más adelante.

c) Un tipo aparte, aunque relacionado con los dos anteriores, es el de "El otro", de Borges. Aquí, 1 y 2 son diferentes etapas cronológicas de un mismo individuo, que coinciden en un tiempo y un espacio dados. El narrador, 1 (que se llama "Jorge Luis Borges"), se encuentra con un joven cuya apariencia y voz le son extrañamente familiares, y que comparte los primeros años de su experiencia personal. Se trata, entonces, de dos seres físicamente independientes, pero que representan dos etapas de la misma personalidad.

En cuanto a su origen, los dobles pueden ser "dados" o "creados". ¿Qué significa esto? El doble "creado" es, generalmente, fabricado por 1. Es lo que ocurre en el caso de "The Dummy", en el que 1, con todos los auxilios de la tecnología moderna (con claros tintes de ciencia-ficción), manufactura un autómata a su imagen y semejanza. Este, 2, habrá de ocupar su lugar y sustituirlo en todas las tareas cotidianas pára que 1 , liberado del peso de sus obligaciones laborales y familiares, pueda llevar libremente la clase de vida que se le antoja. Aqui tampoco puedo dejar 
de mencionar, aunque sólo sea de paso, a Stevenson: recordemos que el Dr. Jekyll se fabrica su segunda personalidad con ayuda de la ciencia.

Pero éstos no son los dobles más frecuentes. En la mayoría de los casos, el personaje 1 se encuentra, como Narciso, con la presencia de 2, en forma más o menos intempestiva. El otro no estaba ahí antes, sino que en un momento dado su presencia se hace patente $y$, como dice el narrador de "Monsieur du Miroir", la relación surge "without any choice on my part". ${ }^{2}$ El hecho de que la aparición de 2 sea independiente de la voluntad de 1 parece ser una característica común de los textos en que hay dobles “dados". Borges 1 no busca la presencia de su alter ego joven, sino que se encuentra con él, un buen día, en una banca de un parque. ${ }^{3}$ William Wilson 2 aparece en la escuela donde estudia 1, pero no había formado parte de su vida anterior; su existencia no sólo no había sido deseada por 1 , sino que le provoca un profundo desagrado. La lejana del cuento de Cortázar es para 1 una presencia mental indefinida que sólo la acompaña desde hace algún tiempo, y que representa una gran molestia, una especie de dolor de cabeza, un malestar que seguramente, dice, se debe a que "la soltería me dañaba, nada más que eso, tener veintisiete años y sin hombre". 4 Y el guerrero moteca de "La noche boca arriba" es interpretado por 1 como un sueño, un delirio provocado por la fiebre y la anestesia.

Todo ello tiene como consecuencia que la aparición de 2 , en los casos de dobles "dados", provoque en I una serie de sentimientos negativos, que pueden llegar al odio ("William Wilson"), al rechazo ("Lejana"), al franco miedo ("La noche boca arriba") 0 , por lo menos, a una inquietud profunda que hace evitar el reencuentro ("El otro"). Pero en todos los casos, sea cual fuere el sentimiento preciso de 1, se presenta en él o en ella una especie de inseguridad, más o menos profunda, que lo lleva a querer resolver la situación, es decir, a querer terminar con ella.

La presencia de 2 no es, no puede ser agradable para 1. En algunos casos, como el de "William Wilson", esta presencia provoca un verdadero delirio de persecución, que es la causa de que 1 quiera destruir a 2. Lo logra finalmente, y termina por asesinarlo, sólo para darse cuenta de que la muerte de 2 significa, inevitablemente, la suya propia. La protagonista de "Lejana" también quiere destruir a la otra ("se doblegará si realmente soy yo", dice cuando piensa en ir a buscarla). ${ }^{5}$ Sin embargo, lo que obtendrá no será una victoria, sino una suplantación. La que triunfa es la otra, que usurpa el lugar de 1 y así, en cierta forma, la destruye. El personaje de Borges, en cambio, elude el conflicto al evitar volver al sitio del encuentro. Por su parte, el narrador/protagonista de "Monsieur du Miroir", aunque no hace nada por destruir a su doble-reflejo, sí reflexiona, con cierto tono de hastío, sobre el hecho de que nunca podrá librarse de él, pues está presente en todos los momentos de su vida, incluso en los más inesperados.

2 N. Hawthorne, “Monsieur du Miroir”, p. 281.

3 Esta banca, por otra parte, constituye un ejemplo de objeto doble: para 1 está situada en Cambridge; para 2, en Ginebra.

4 J. Cortázar, "Lejana", p. 46.

5 Ibid., p. 47. 
La conclusión lógica de este desagrado es que el desenlace de los cuentos de dobles suele mostrar el triunfo de uno u otro de los protagonistas, triunfo que no es sencillo, sino que va acompañado, implícita o explícitamente, de la destrucción del otro.

Otro punto en común que comparten los distintos dobles, sean dados o creados, es que el hecho de formar parte de una de esas extrañas parejas constituidas por 1 y 2 no es una cuestión de esencia, sino un estado. Los personajes se fabrican un doble o se encuentran con él, pero no son dobles desde su origen sino que, a partir de cierto momento, están en dicha situación. Claro que esto se refiere tan sólo a 1 y nunca (o casi nunca) a 2. Es evidente que 1 no tenía un doble (o no sabía que lo tenía) antes de la entrada en escena de 2 . La excepción en este caso, por razones obvias, es "Monsieur du Miroir": "It is my belief", dice el narrador, "that we came into existence together".6 Esto no puede causar extrañeza si recordamos la naturaleza de 2: Monsieur du Miroir, como su nombre lo indica, es la imagen de 1 en el espejo.

Algo muy especial ocurre con este estado de doble: aunque en un principio, como hemos visto, el tener un doble no es cuestión de esencia, una vez que éste aparece, la relación entre él y su original, la relación entre 1 y 2 , se vuelve indisoluble, permanente y esencial. Su disolución significa la muerte. Ya indiqué antes que la muerte de William Wilson 2 significa la destrucción de 1. Esto vale también en el caso de los otros dobles: para que Monsieur du Miroir deje de ser visible, 1 tiene que morir al mismo tiempo que él, puesto que 2 no es más que su reflejo. Al intercambiarse con 1 la lejana de Budapest, ¿cuál de las dos sería la que moriria si una de ellas llegase a desaparecer? Si el joven Borges 2 es eliminado, es lógico pensar que Borges 1, que es su secuela en el tiempo, fatalmente tiene que desaparecer, como consecuencia inmediata. La muerte del guerrero moteca, el 2 de "La noche boca arriba", significa que 1 , el motociclista, ha muerto también. El 1 de "The Double", que creó el autómata porque su vida le era insoportable, no podría resistir la desaparición de 2 (de hecho, cuando 2 decide que él tampoco quiere esa vida, la única alternativa posible para 1 es crear un segundo autómata, con lo cual nos encontramos ante la presencia de un 3). Así pues, lo que originalmente era un estado se transforma aqui, por el hecho mismo de su existencia, en una esencia.

Ahora bien, el lector se entera de todas estas cosas en una forma notablemente parcial. Ocurre que, en todos los textos, la visión del fenómeno se da exclusivamente desde un punto de vista, que siempre es el de 1 . Sea cual sea el tipo de narrador: en primera persona ("El otro", "The Dummy" y "William Wilson"), narrador impersonal ("La noche boca arriba"), o combinación de primera persona con narrador impersonal ("Lejana"), siempre vemos las cosas a través de los pensamientos, las sensaciones y las emociones de 1 . Desde este aspecto, 2 no deja de ser, auténticamente, una sombra, una figura que está más o menos en el fondo, que nunca habla por sí misma ni de sí misma; nunca se encuentra verdaderamente en el

6 N. Hawthorne, op. cit., p. 285. 
primer plano de la historia, aunque pueda ocupar el primer plano de los pensamientos de 1 y llegar a convertirse en una verdadera obsesión. De este modo la visión del lector, extrañamente, será siempre fragmentada, siempre parcial. Esto vale aun en el caso de "La noche boca arriba", en que el narrador a veces relata los pensamientos de 2 , pero sin perder nunca la conciencia de que esta segunda personalidad es un aspecto delirante y enfermo de la primera, y está supeditada a ella (salvo, claro, en la sorpresa final). En "El otro", Borges 2 toma varias veces la palabra, pero la historia es contada por 1. "Lejana" es narrada por medio del diario de 1 y al final interviene, por el espacio de breves líneas, un narrador impersonal que ve las cosas desde afuera. Esta no participación directa de 2 en la historia ocurre invariablemente, y justifica el hecho de que ocupe precisamente el segundo lugar. Nunca se pierde realmente la conciencia de quién es 1 y quién 2, quién llegó primero y quién después, quién es el individuo y quién su sombra.

Esta visión fragmentaria de los dobles explica, en parte, otra característica común de los textos que nos ocupan: el secreto. La existencia de los dobles, en efecto, no suele ser conocida por los otros. Así, nunca puede ver el lector qué efecto tiene este fenómeno en los demás personajes del cuento, que no pasan de ser meras comparsas que entran y salen esporádicamente, sin intervenir de manera significativa en la relación entre 1 y 2 . En algunos casos (y "William Wilson" es un ejemplo de esto), hasta la misma identidad física de los personajes pasa completamente desapercibida para los demás.

Antes de terminar quiero señalar un rasgo más, cuya presencia me parece notable, pero para el cual no he podido encontrar una explicación convincente: se trata de los problemas presentados por el espacio (y el tiempo, claro, pero hablaré tan sólo del espacio). Se presentan transgresiones de tipo espacial en todos estos cuentos, pues parecería que hay, para los dobles, una imposibilidad de coincidir en el mismo espacio. Veamos: en "El otro", 1 y 2 están aparentemente sentados en la misma banca del parque; sin embargo, 1 se encuentra en Cambridge y 2 en Ginebra (y además, hay un desfasamiento temporal, pues para 1 los hechos ocurren en febrero de 1969, mientras que 2 se encuentra en 1918). Ninguno de los dos pasa del lado del otro, y ni siquiera se tocan. Es cierto que hay un intercambio de objetos, pero no los conservan, los devuelven, como si la vida no pudiera soportar esa permanencia de una cosa en un tiempo que le es ajeno. Los personajes de "La noche boca arriba" están a miles de kilómetros uno del otro y separados por una distancia de varios siglos. Cuando 2 no puede volver a transformarse en 1, en su cama de hospital, es decir, cuando la dimensión espacial se reduce, sobreviene la muerte. En "Lejana", 1 vive en Buenos Aires y 2 en Budapest; cuando se encuentran en un mismo lugar es cuando ocurre la crisis. El caso que parece más sencillo y lógico es el de Monsieur du Miroir, pues es obvia la razón por la que los dos personajes no pueden coincidir del mismo lado del espejo. Cuando William Wilson 1 logra por fin acorralar a 2 en el mismo espacio que él por más de un instante, lo mata. $Y$ en cuanto a "The Dummy", 1 acompaña a 2 en múltiples momentos de su vida, pero ocurre entonces un fenómeno muy especial: es que 1 parece volverse enteramente invisible, aunque esto no se explicite en el texto. Es cierto que, al final del cuento, 1 
visita a 2 y a 3 , pero en ese momento han dejado de ser auténticamente dobles, pues el físico y la personalidad de 1 han variado sensiblemente. 1 ya no es el que era al comienzo, y en este sentido, también en su caso se puede hablar de una destrucción. 2 y 3 son los únicos que permanecen, los únicos sobrevivientes verdaderos, y ellos no se encuentran entre sí.

Los dobles se presentan, como hemos podido observar a través de estas pocas muestras, en formas harto diferentes. Quedaria por ver, desde luego, cuál es la función que cumplen y cuál su significado verdadero. Esto, sin embargo, es la segunda parte de mi estudio, que permitirá, espero, llegar a ciertas conclusiones más o menos claras y definitivas. Y digo más o menos, porque el motivo del doble, como todos los demás motivos literarios, evoluciona con el tiempo y pasa por transformaciones incesantes. Espero que, con el tiempo, este estudio también evolucione y llegue a una forma más completa.

\section{BIBLIOGRAFIA}

BORGES, Jorge Luis. "El otro", en El libro de arena. Buenos Aires, Emecé, 1975. CORTÁZAR, Julio. “Lejana”, en Bestiario. Buenos Aires, Sudamericana, 1968.

_- - ."La noche boca arriba", en Final del juego. Buenos Aires, Sudamericana, 1968.

HAWTHORNE, Nathaniel. "Monsieur du Miroir", en The Complete Short Stories of Nathaniel Hawthorne. New York, Hannover House, 1959.

'OE, Edgar Allan. "William Wilson", en The Complete Tales and Poems of Edgar Allan Poe. New York, Random House, 1938.

2ANK, Otto. The Double, a Psychoanalytic Study. Ed. y trad. de Harry Tucker, Jr. Chapel Hill, University of North Carolina Press, 1971.

iONTAG, Susan. "The Dummy", en I, etcetera. New York, Random House, 1979. 